\title{
Das AGUST-Projekt
}

\section{Analyse von Gemeinsamkeiten und Unterschieden schulspezifischer Psychotherapieverfahren}

\author{
Cornelia Stegmann, Rosmarie Barwinski, Katrin Hartmann, Mario Schlegel \& Agnes von Wyl
}

\author{
Psychotherapie-Wissenschaft 9 (2) 74-84 2019 \\ www.psychotherapie-wissenschaft.info \\ CC BY-NC-ND \\ https://doi.org/10.30820/1664-9583-2019-2-74
}

\begin{abstract}
Zusammenfassung: Die Wissenschaftskommission der Schweizer Charta für Psychotherapie (WiKo), ein Organ der Assoziation Schweizer Psychotherapeutinnen und Psychotherapeuten (ASP), hat mit dem AGUST-Projekt das Vorhaben umgesetzt, Gemeinsamkeiten und Unterschiede in der Anwendung von psychotherapeutischen Interventionen zu untersuchen. Dafür wurden 24 Vertreter acht verschiedener Psychotherapieschulen zu einem APA-Lehrvideo befragt. In drei Forschungskolloquien, die zwischen September 2018 und Januar 2019 stattfanden, wurde das Video in Arbeitsgruppen von ihnen bewertet. Am Beispiel der Klientin des Videos wurden zudem alternative psychotherapeutische Interventionsvorschläge und Therapieziele in den Gruppen erarbeitet sowie deren Begründungen erfasst. Im Mittelpunkt des Forschungsinteresses stand dabei das konkrete Vorgehen der einzelnen Schulen. Dabei zeigten sich Gemeinsamkeiten und Unterschiede zwischen den einzelnen Arbeitsgruppen. Bei der Bewertung des Videos, der alternativen Reaktionsvorschläge und deren Zuordnung mittels PAP-S-Rating-Manual unterschieden sich die Gruppen weniger als bei der Begründung der alternativen Interventionen, ihrer Beschreibung der Probleme der Klientin des Videos und den Begrifflichkeiten zur Abbildung ihres schulspezifischen Hintergrundes.
\end{abstract}

Schlagwörter: Psychotherapieforschung, psychotherapeutische Interventionen, Wirkfaktoren, Psychotherapieschulen

\section{Hinführung zum Thema}

Prof. Bernhard Strauss stellte 2017 bei einer Gastvorlesung an der Zürcher Hochschule für Angewandte Wissenschaften (ZHAW), Department Angewandte Psychologie, die Vermutung an, dass es in 30 Jahren eine «Einheitstherapie» geben wird. Es ist vorstellbar, dass sich die Psychotherapie der Zukunft zusammensetzen wird aus den unterschiedlichen schultheoretischen Konzepten, wie sie gegenwärtig bestehen und vereinheitlicht angeboten werden könnte. Personen die psychotherapeutische Unterstützung suchen, würden dann im optimalen Fall eine Therapie erhalten, die wirksame Prozesse verfolgt und alle Faktoren berücksichtigt, die zu ihrem Therapieerfolg beitragen. Es ist anzunehmen, dass die schultheoretischen Ausrichtungen unterschiedliche Begründungen zur Auswahl der entsprechenden Prozesse und Faktoren verwenden, die gewählten konkreten Interventionen aber ähnlich sind. Die Psychotherapieforschung geht heute von schulenübergreifend wirksamen Verfahren aus und stellt die Interaktion zwischen KlientIn und TherapeutIn für den erfolgreichen Verlauf einer Behandlung in den Vordergrund. Der Einfluss der jeweiligen Schulen scheint dabei jedoch nicht entscheidend zu sein.

Gegenwärtig zeichnet eine breite Diversität die schweizerische Psychotherapielandschaft aus, der Assoziation Schweizer Psychotherapeutinnen und Psychotherapeuten (ASP) sind 25 Weiterbildungsinstitute angeschlossen. Diese Vielfalt ist historisch gewachsen und traditionell verankert und entspringt dem Gedanken der individuellen Passung zwischen KlientIn und TherapeutIn bzw. der Therapieform. Das Angebot unterschiedlicher Formen von Psychotherapie entspricht vermutlich den verschiedenen Bedürfnissen der KlientInnen und ihren Erwartungen an eine Therapie. Genauso wie es auf TherapeutInnenseite unterschiedliche Präferenzen bezüglich der schultheoretischen Konzepte gibt. Durch die bestehende Vielfalt innerhalb der schweizerischen Psychotherapielandschaft wird den unterschiedlichen Ansprüchen von KlientInnen und PsychotherapeutInnen Rechnung getragen. Seit ihren Anfängen setzte sich die Psychotherapie mit der Erforschung der Wirkungsweise ihrer Heilmethode auseinander. Durch die Nähe zur Psychiatrie wurde sie stark durch medizinische Paradigmen geprägt, was in der aktuellen wissenschaftlichen Auseinandersetzung auch durchaus kritisch betrachtet wird. Die verschiedenen Therapierichtungen, die sich im Laufe der Zeit gebildet haben, sind mit unterschiedlichen theoretischen Konzepten ausgestattet und ziehen unterschiedliche Erklärungsmodelle für den Erfolg ihrer psychotherapeutischen Interventionen heran. Unter dem sogenannten «Schulenstreit» lag der wissenschaftliche Fokus auf der Legitimation einzelner therapeutischen Ausrichtungen als wirkungsvolle Behandlung psychischer Leiden. Eine differenziertere und aktuellere Perspektive zur Erklärung von erfolgreicher Psychotherapie stellt die Wirkfaktorenforschung dar. Für eine Übersicht werden dazu im Folgenden die wichtigsten Erkenntnisse aus 


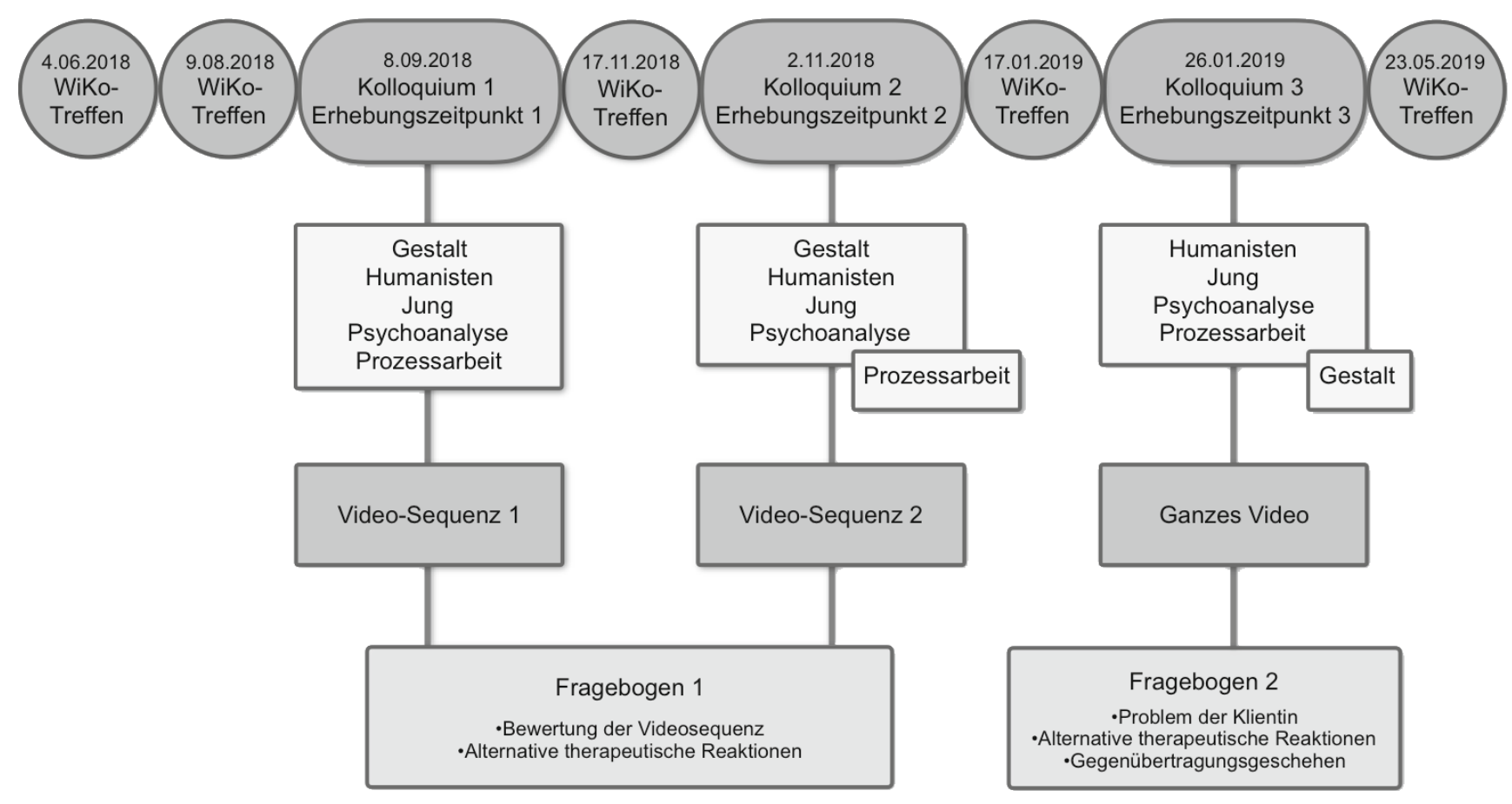

Abb. 1: Übersicht über den AGUST-Projektverlauf

diesem Gebiet aufgezeigt. Beim AGUST-Projekt steht die konkrete psychotherapeutische Intervention, im Sinne der verbalen Reaktion auf die Erzählungen der KlientInnen, im Forschungsinteresse. Die vorliegende Arbeit soll die wissenschaftliche Begleitung des Projektes aufzeigen und möchte damit einen Beitrag zur aktuellen Psychotherapieforschung leisten.

\section{Das AGUST-Projekt}

Das AGUST-Projekt entstand durch eine Initiative der vier Mitglieder der Wissenschaftskommission (WiKo) der ASP. AGUST ist das Akronym für: Analyse von Gemeinsamkeiten und Unterschieden von schulspezifischen (Psycho-) Therapieverfahren. Auch fachlich wurde das Projekt von der WiKo begleitet, die bereits die Praxisstudie ambulante Psychotherapie Schweiz (von Wyl et al., 2016) initiierte und begleitete. In dieser Studie wurden unterschiedliche psychotherapeutische Behandlungsmethoden miteinander verglichen. Dazu wurde auch ein Rating-Manual entwickelt, um therapeutische Interventionen objektiv einschätzen zu können: Das Rating-Manual zur objektiven Einschätzung therapeutischer Interventionen von Psychotherapeuten unterschiedlicher schultheoretischer Konzepte (PAP-S-RM).

Das AGUST-Projekt bezieht sich auf diese Praxisstudie: Nachdem jedoch in der Praxisstudie die tatsächlichen Interventionen in den Psychotherapiestunden verschiedenen schulenspezifischen Interventionen zugeordnet wurden, suchte das AGUST-Projekt nun nach Gemeinsamkeiten und Unterschieden einerseits in der Theorie und andererseits in der Anwendung unterschiedlicher schultheoreti- scher Konzepte. Um möglichst gegenständlich auf den psychotherapeutischen Alltag bezogen zu sein, ging das Projekt dabei von konkreten Interventionen aus. Anhand einer auf Video aufgenommenen Therapiestunde diskutierten TherapeutInnen verschiedener Therapieschulen in drei Forschungskolloquien der ASP die Anwendung konkreter psychotherapeutischen Interventionen.

Ambühl \& Orlinsky (1997) haben Unterscheidungen hinsichtlich der angestrebten Ziele von PsychotherapeutInnen mit unterschiedlichen Ausrichtungen untersucht. Sie fanden im Vergleich von analytisch-psychodynamischer und humanistischer Orientierung grosse Unterschiede bezüglich der Erklärung der Entstehung und Veränderbarkeit psychischer Störungen und grosse Übereinstimmung bezüglich der Ziele der Behandlung. Das legt den Schluss nahe, dass sich die einzelnen theoretischen Konzepte der TherapeutInnen voneinander abheben, die praktische Vorgehensweise jedoch ähnlich sein könnte.

Im AGUST-Projekt wurden die theoretische und die anwendungsbezogene Perspektive aufgegriffen und die konkrete Intervention innerhalb der psychotherapeutischen Behandlung fokussiert. Dafür wurden drei Treffen innerhalb der Forschungskolloquien der ASP genutzt (Übungs-, Vertiefungs- und Theoriekolloquium). Hier treffen sich quartalsweise die VertreterInnen der verschiedenen Therapieschulen. Für einen Überblick zum gesamten Projektverlauf siehe Abbildung 1. Zur Annäherung an das Vorgehen in der Praxis wurde im AGUST-Projekt die gemeinsame Videoanalyse verwendet. Anhand einer exemplarischen Psychotherapiesitzung konnten die Teilnehmenden Rückschlüsse zu ihrer eigenen therapeutischen Tätigkeit ziehen und durch konkrete Interventionsvorschläge ihr therapeutisches Arbeiten darstellen. 


\section{Definition der Begrifflichkeiten}

Psychotherapie wird von Strotzka (1969, S. 32) folgendermassen definiert:

\begin{abstract}
«Psychotherapie ist ein bewusster und geplanter interaktioneller Prozess zur Beeinflussung von Verhaltensstörungen und psychischen bzw. psychosomatischen Leidenszuständen, die in einem Konsensus (möglichst zwischen Patient, Therapeut und Bezugsgruppe) für behandlungsbedürftig gehalten werden, mit psychologischen Mitteln (durch Kommunikation), meist verbal, aber auch averbal, in Richtung auf ein definiertes, nach Möglichkeit gemeinsam erarbeitetes Ziel (Symptomminimalisierung und/oder Strukturänderung der Persönlichkeit) mittels lehrbarer Techniken auf der Basis einer Theorie des normalen und pathologischen Verhaltens. In der Regel ist dazu eine tragfähige emotionale Bindung notwendig.»
\end{abstract}

Diese 50 Jahre alte Umschreibung Strotzkas besitzt immer noch Allgemeingültigkeit und lässt sich auf alle therapeutischen Ausrichtungen anwenden. Er beschreibt darin die interaktionale Prozesshaftigkeit sowie die therapeutische Beziehung, die auch in jüngster Zeit wieder in den Mittelpunkt des Forschungsinteresses gerückt ist (Strauss \& Schauenburg, 2017; Norcross \& Lambert, 2018). Im Zusammenhang mit dem kontextuellen Metamodell beschreiben Wampold und Kollegen (2018, S. 86) Psychotherapie als «soziale Heilpraxis, die sich die Gesamtheit der menschlichen Intelligenz zunutze macht.» Die Autoren gehen dabei von der Annahme aus, dass der Mensch als soziales Wesen mit sozialen Mitteln geheilt werden kann.

Psychotherapeutische Interventionen werden als «Massnahme, deren Wirkung auf ein Zielmerkmal untersucht wird» (Wirtz, 2016) beschrieben, als «klinische Strategien» (Goldfried, 1980), «Wirkprinzipien» (Grawe, 2000) oder «Techniken» (WBP, 2008). Diese Begrifflichkeiten beschreiben unterschiedliche Perspektiven und transportieren mitunter unterschiedliche Inhalte. Die Unterscheidung der psychotherapeutischen Interventionen in allgemeine und spezifische Faktoren hat sich in der Forschungswelt dennoch etabliert und wird in der vorliegenden Arbeit weiter aufgegriffen. Pfammatter \& Tschacher (2012, S. 69) definieren allgemeine und spezifische Wirkfaktoren folgendermassen:

«Allgemeine Wirkfaktoren: Therapievariablen, die implizit in allen Psychotherapieverfahren auftreten und zu therapeutischen Veränderungen beitragen. Sie sind inhärenter Bestandteiler jeglicher Form von Psychotherapie. Sie kommen weder exklusiv nur bei bestimmten Psychotherapiemethoden zum Tragen noch spielen sie in der Behandlung spezifischer psychischer Störungen eine besondere Rolle.

Spezifische Wirkfaktoren: Die in einem bestimmten Psychotherapiekonzept explizit verankerten Therapietechniken. Spezifisch bezieht sich dabei sowohl auf die besondere Bedeutung eines Wirkfaktors für ein bestimm- tes Psychotherapie- oder Störungsmodell, als auch auf seine explizite theoretische Spezifizierung im Rahmen dieses Therapie- oder Störungskonzepts. Spezifische Wirkfaktoren sind einerseits Standardtechniken der verschiedenen Psychotherapieschulen [...] andererseits fallen darunter auch störungsspezifische psychotherapeutische Vorgehensweisen».

Gleichzeitig werden spezifische Techniken realisiert und ihre Unterscheidung wird dadurch hinfällig. Sie begründen das damit, dass allgemeine Wirkfaktoren ähnliche Muster von Zusammenhängen aufweisen und deshalb mit spezifischen Techniken zusammengefasst werden können. In einem Taxonomieprojekt (Pfammatter \& Tschacher, 2016) konnten sie vier Cluster von Zusammenhängen allgemeiner Wirkfaktoren mit spezifischen Techniken nachweisen. Allgemeine Faktoren lassen sich demnach auf die Klassen kognitive Verarbeitung, Problembewältigung, emotionale Verarbeitung und Beziehungs- und Motivationsaufbau beziehen.

Eine andere Herangehensweise um das psychotherapeutische Vorgehen innerhalb einer Sitzung zu beschreiben, sind neben den Interventionen die Ziele (goals, targets, intentions), mit deren Hilfe die Interventionen umgesetzt werden sollen. Hill und O'Grady $(1985$, S. 3) definieren diese Ziele folgendermassen:

"Intentions represent what the therapist wants to accomplish through his or her behaviour within the session. An intention is the cognitive component that mediates the choice of intervention. Intentions refer to why, whereas interventions or techniques refer to what the therapist does.»

Demzufolge dienen die Ziele der TherapeutInnen der Begründung und Auswahl ihrer angewendeten Interventionen. Werden diese Ziele in der Therapie gemeinsam mit KlientInnen festgelegt bzw. besteht ein Konsens über die Ziele, dann trägt dies erwiesenermassen zum Therapieerfolg bei (Norcross \& Lambert, 2018). Der Schwerpunkt beim AGUST-Projekt lag jedoch bei der Zielformulierung der TherapeutInnen in Abhängigkeit ihrer jeweiligen theoretischen Ausrichtung.

\section{Herleitung der Forschungsfragen}

In der Psychotherapie wird das Gespräch als Heilbehandlung, als talking cure, angeboten. Die Interaktions- und Kommunikationsprozesse, die nach Wampold und Kollegen (2018) die sozialen Mittel darstellen, mit denen Menschen geheilt werden können, lassen sich unterschiedlich gestalten. Die einzelnen Ausrichtungen in der Psychotherapie legen dabei unterschiedliche Schwerpunkte. Sie vertreten unterschiedliche Perspektiven, die jeweilige Theorie wird unterschiedlich vermittelt. Das Ausmass der Anwendung des schultheoretischen Wissens muss nicht ausschlaggebend für den Therapieerfolg sein. Betrachtet man die Konzepttreue (adherence), also wie 


\begin{tabular}{|l|l|l|}
\hline \multicolumn{1}{|c|}{$\begin{array}{c}\text { AGUST-Arbeitsgruppen } \\
\text { Anzahl d. Teilnehmenden) }\end{array}$} & \multicolumn{1}{|c|}{ Methode } & \multicolumn{1}{c|}{ Organisation } \\
\hline $\begin{array}{l}\text { Gestaltpsychotherapie } \\
\text { (1 Person) }\end{array}$ & Gestalttherapie & $\begin{array}{l}\text { Schweizer Verein für Gestalttherapie und Integrative } \\
\text { Therapie (SVG) }\end{array}$ \\
\hline $\begin{array}{l}\text { Humanistische Psychotherapie } \\
\text { (4-6 Personen) } \\
\text { Existenzanalyse } \\
\text { Körperpsychotherapie }\end{array}$ & $\begin{array}{l}\text { Existenzanalytische Therapie } \\
\text { Integrative Body Psychotherapy } \\
\text { Gespräch, Focusing, Körper (GFK) }\end{array}$ & $\begin{array}{l}\text { Institut für Integrative Body Psychotherapy (IBP) } \\
\text { Ausbildungsinstitut Gespräch, Focusing, Körper (GFK) }\end{array}$ \\
\hline $\begin{array}{l}\text { Jung'sche Psychoanalyse } \\
\text { (4-6 Personen) }\end{array}$ & Analytische Psychologie nach Jung & C.G. Jung-Institut Zürich \\
\hline $\begin{array}{l}\text { Freud'sche Psychoanalyse } \\
\text { 5-6 Personen) } \\
\text { Daseinsanalyse }\end{array}$ & Analytische Psychologie nach Freud & Psychoanalytisches Seminar Zürich (PSZ) \\
\hline $\begin{array}{l}\text { Prozessorientierte Psychotherapie } \\
\text { (2-3 Personen) }\end{array}$ & $\begin{array}{l}\text { Prozessorientierte Psychotherapie } \\
\text { nach Mindell }\end{array}$ & Schweizerische Gesellschaft für Daseinsanalyse (DaS) \\
\hline
\end{tabular}

Tab. 1: Zusammensetzung der AGUST-Arbeitsgruppen

stark sich PsychotherapeutInnen dem eigenen schultheoretischen Konzept verpflichtet fühlen, dann lässt sich kein signifikanter Einfluss auf das Therapieergebnis erkennen (Tschuschke et al., 2014; Tschuschke, 2016). Im AGUST-Projekt wird davon ausgegangen, dass die Abgrenzung zwischen den einzelnen Therapiekonzepten auf theoretischer Ebene besteht. Konkret sollen folgende Fragestellungen beantwortet werden: Wie - bzw. nicht unterscheidet sich das konkrete praktische Vorgehen in Psychotherapien in Abhängigkeit der zugrundeliegenden theoretischen Konzepte der einzelnen Schulen? Wo zeigen sich Gemeinsamkeiten und wo zeigen sich Unterschiede in der Beurteilung, bei der Auswahl von Alternativinterventionen und der Gesamtevaluation des Videos durch PsychotherapeutInnen unterschiedlichen schultheoretischen Hintergrundes?

\section{Teilnehmende}

An den Forschungskolloquien des AGUST-Projektes bzw. zu den Erhebungszeitpunkten (EZ) nahmen 17 (EZ 1), 19 (EZ 2) und 22 (EZ 3) PsychotherapeutInnen bzw. Mitglieder der ASP teil, darunter auch die Mitglieder der WiKo. Über alle Erhebungszeitpunkte betrachtet haben 14 Frauen und zehn Männer am AGUST-Projekt mitgewirkt.

Zu Beginn des Einführungs- und Übungskolloquiums (EZ 1) wurden folgende Arbeitsgruppen gebildet, die aufgrund ihrer historisch gewachsenen Grundausrichtung eingeteilt wurden: Gestaltpsychotherapie, Humanistische Psychotherapie (mit den Therapierichtungen Existenzanalyse und Körperpsychotherapie) Jung'sche Psychoanalyse, Freud'sche Psychoanalyse und Prozessorientierte Psychotherapie (zur Zusammensetzung s. Tab. 1). Die
Arbeitsgruppe Gestaltpsychotherapie wurde durch einen Teilnehmer vertreten und stellt somit keine Gruppe im eigentlichen Sinne dar. Dadurch, dass im AGUST-Projekt aber möglichst viele unterschiedliche Therapieschulen abgebildet und miteinander verglichen werden sollten, wurde Gestaltpsychotherapie als eigene Arbeitsgruppe behandelt. Zur Beantwortung der Fragestellung wurden 15 ExpertInnen-Gruppendiskussionen geführt, verschriftlicht und ausgewertet. Diese Gruppendiskussion war stellvertretend für ihre jeweiligen Schulen bzw. Institute.

\section{Datengrundlage und Instrumente}

Datengrundlage ist die Aufzeichnung der Diskussionen zur Videoanalyse der Arbeitsgruppe aus den Forschungskolloquien; ausserdem deren schriftliche Ergänzungen zwischen den Kolloquien und die erhobenen Aussagen der Schulen ausserhalb der Forschungskolloquien. Die Gruppendiskussionen orientierten sich dabei an zwei Fragebogen, die innerhalb der WiKo für das AGUSTProjekt erstellt wurden.

Fragebogen 1 behandelt im ersten Teil die Bewertung der psychotherapeutischen Intervention aus dem Video, es wird nach den vermuteten Interventionen und Zielen der Therapeutin (Norka Malberg) gefragt. Im zweiten Teil von Fragebogen 1 stehen die möglichen alternativen Reaktionsvorschläge der Arbeitsgruppen im Mittelpunkt. Die Fragen beziehen sich wieder auf Interventionen und Ziele, also darauf, was die ProbandInnen tun würden, wären sie anstelle von Frau Malberg die TherapeutInnen. Dies wird ergänzt durch eine Frage nach der konkreten Intervention, ihrer wörtlichen Formulierung und der Begründung ihres Einsatzes. Abschliessend werden die Arbeitsgruppen gebeten, ihre Alternativvorschläge mit 
dem PAP-S-RM (Tschuschke et al., 2014) zu codieren. $\mathrm{Zu}$ beachten ist, dass zwei verschiedene Filmsequenzen gezeigt wurden. Zu EZ 1 der Ausschnitt bis Min. 2:57, zu EZ 2 bis Min. 5:23. Die beiden Sequenzen werden nachfolgend vorgestellt.

Fragebogen 2 kam zum dritten Erhebungszeitpunkt, dem Theoriekolloquium, zum Einsatz. Hier wurde der ganze Film visioniert und dazu Stellung genommen, wieder bezüglich Alternativinterventionen und Zielen. Wie würden die einzelnen Schulen in einem solchen APA-Video ihre Konzepte darstellen? Da die vermuteten Probleme der Klientin dies beeinflussen könnten, wurden sie ebenfalls abgefragt. Der zweite Fragebogen wurde ausserdem mit der Thematisierung der Gegenübertragungsgefühle der ProbandInnen gegenüber der Klientin (Karina) ergänzt. Diese könnten ebenfalls eine Rolle spielen für die Auswahl der alternativen therapeutischen Reaktionen der ProbandInnen.

Für die Auswertung wurde die qualitative Inhaltsanalyse nach Mayring (2016) eingesetzt und unterstützend mit der MAXQDA-Software gearbeitet. Daneben kam das PAP-S-RM zur Anwendung, um die Bewertungen der Interventionen aus dem Video zu erfassen und die konkreten Alternativinterventionen der Arbeitsgruppen zuteilen zu können. Zudem wurden von der Autorin der vorliegenden Arbeit die Abbildungen des schulspezifischen Hintergrundes der Gruppen mittels PAP-S-RM codiert. Die einzelnen Arbeitsgruppen haben ebenfalls mittels PAPS-RM ihre eigenen Alternativinterventionen eingeteilt. Für ein Fremdrating der Videosequenzen hat sich Frau Koemeda-Lutz (SGBAT) freundlicherweise zur Verfügung gestellt. Sie hat massgeblich bei der Entwicklung des PAP-S-RM beigetragen und damals unter anderem die Schulung der Rater umgesetzt.

Das Video wird von der APA auf ihrer Website als Demonstration der Wirksamkeit der MB-Therapie durch Dr. N. Malberg beschrieben. ${ }^{1}$ Bei der Klientin handelt es sich laut APA-Angaben um Karina, eine junge Frau, die mit Ablösungsschwierigkeiten zu tun hat und eine gesunde Beziehung mit ihrer Mutter etablieren möchte. Das Gespräch im Film wurde in Originalsprache gezeigt, amerikanischem Englisch, mit englischen Untertiteln. Den Teilnehmenden und Frau Koemeda-Lutz stand vor dem Start des AGUST-Projektes eine Abschrift der Untertitel zur Verfügung. Vor dem Theoriekolloquium (EZ 3) wurde den Teilnehmenden ausserdem das Video gegeben. Mit welchem schultheoretischen Hintergrund Frau Malberg arbeitet, wurde vor dem Übungskolloquium ausserhalb der WiKo nicht thematisiert. Das Therapiegespräch dauert 41:30 Minuten. Die Begegnung zwischen Klientin und Therapeutin beschränkt sich auf das aufgezeichnete Gespräch, dem ein Telefonat zwischen Therapeutin und Klientin vorausgegangen ist. Weitere Treffen wurden nicht vereinbart. Unter welchen (finanziellen) Bedingungen die Klientin an diesem Therapiegespräch teilnimmt, ist nicht klar.

Sequenz 1 umfasst das Gespräch bis zu Min. 2:57. Darin fragt Frau Malberg Karina: «If your mom was here with us, what do you think she would say about that?

1 https://www.apa.org/pubs/videos/4310961 (20.05.2019).
How would she understand it? Would she understand it differently you think?» Auf diesen Ausschnitt im Sinne einer psychotherapeutischen Intervention wird im Fragebogen zum EZ 1 Bezug genommen. Nach Absprache mit Frau Koemeda-Lutz wurde Sequenz 1 mit der PAP-S-RMNr. 100 als zirkuläre Frage codiert. Zirkuläres Fragen ist der systemisch-orientierten Psychotherapie zuzuordnen und gilt daher als spezifischer Faktor.

Zum EZ 2 wurde Sequenz 2 präsentiert, die mit Frau Malbergs Frage bei Min. 5:23 endet: «So, what - does she let you know when there's disappointment or do you immediately feel it in yourself even when she doesn't let you know?» Hier wurde Nr. 8, Arbeit am subjektiven Erleben/Empfinden, mit dem PAP-S-RM codiert. Diese Intervention lässt sich den allgemeinen Wirkfaktoren zuordnen. Den Teilnehmenden wurden beide Fremdratings erst zu EZ 3 mitgeteilt.

\section{Auswertungsmethoden}

Zur Beantwortung der Fragestellungen wurde wie bereits erwähnt das PAP-S-RM verwendet, sowie mit der qualitativ orientierten kategoriengeleiteten Textanalyse nach Mayring (2010) gearbeitet. Dafür wurden die Interpretationsregeln zu Paraphrasierung, Generalisierung auf das Abstraktionsniveau und erster und zweiter Reduktion (Mayring, 2015, S. 72) angewendet und wiederholt überprüft.

Die aufgezeichneten Diskussionsbeiträge der Arbeitsgruppen wurden zuerst verschriftlicht und geordnet, das heisst, die Aussagen, die teilweise von der Fragebogenreihenfolge abwichen oder sich überschnitten, wurden den Kategorien aus dem Fragebogen zugeordnet. Für Fragebogen 1 waren das: Intervention, konkrete Intervention, Ziel und Begründung. Mit Fragebogen 2 ist gleich verfahren worden. Hier wurden die Kategorien: Probleme der Klientin, schulspezifische Interventionen und Ziele sowie Gegenübertragungsgeschehen übernommen. Die den Kategorien zugeordneten Texte wurden den Arbeitsgruppen zum Gegenlesen zugestellt und Berichtigungen ihrerseits aufgenommen. Es folgten der Durchgang der Paraphrasierung, Generalisierung und darauf die Reduktion der Texte.

Die induktive Kategorienbildung wurde vereinfacht durch die Anwendung der Klassen (Cluster) allgemeiner Wirkfaktoren von Pfammatter \& Tschacher (2016) als induktive Kategorie innerhalb des abschliessenden Durchgangs des Datenmaterials. Sie liessen sich auf alle Fragebogenkategorien zu den Interventionen und Zielen übertragen und waren hilfreich für den Überblick über die vorliegende Datenmenge.

Für die Kategorie der Begründung der alternativen Intervention wurden die Cluster ausserdem in Zusammenhang gebracht mit den von Pfammatter \& Tschacher (2012, S. 71) recherchierten allgemeinen Wirkfaktoren. Nach einer ersten Auswertung der Begründung zu den möglichen alternativen Reaktionen haben sich verschiedene Schwerpunkte bzw. induktive Kategorien gezeigt. Diese zeigten Ähnlichkeiten mit den Clustern von Pfam- 


\begin{tabular}{|l|l|l|}
\hline \multicolumn{1}{|c|}{ AGUST-Gruppe } & \multicolumn{1}{|c|}{ Videosequenz 1 } & \multicolumn{1}{c|}{ Videosequenz 2 } \\
\hline Gestaltpsychotherapie & $\begin{array}{l}\text { Verbindung zur Klientin kriegen, } \\
\text { eine Beziehung schaffen }\end{array}$ & Klärung, ob Enttäuschung ein Introjekt der Klientin ist \\
\hline $\begin{array}{l}\text { Humanistische } \\
\text { Psychotherapie }\end{array}$ & $\begin{array}{l}\text { Fördern der Perspektivenübernahme und } \\
\text { ein Perspektivenwechsel; Erweitern der } \\
\text { Weltsicht der Klientin }\end{array}$ & $\begin{array}{l}\text { Differenzierung zwischen subjektivem Erleben der Patientin } \\
\text { und dem was objektiv geschieht }\end{array}$ \\
\hline Jung'sche Psychoanalyse & $\begin{array}{l}\text { Ganzheitliche Objektwahrnehmung; } \\
\text { Mentalisierung }\end{array}$ & $\begin{array}{l}\text { Herausfinden, ob die Patientin mentale Vorgänge in sich } \\
\text { selbst, in der Mutter reflektieren kann, auf das hört was } \\
\text { die Mutter sagt, sich etwas einbildet }\end{array}$ \\
\hline $\begin{array}{l}\text { Prozessorientierte } \\
\text { Psychotherapie }\end{array}$ & $\begin{array}{l}\text { (keine Angaben) } \\
\text { Freud'sche Psychoanalyse }\end{array}$ & $\begin{array}{l}\text { Weitere Exploration des Problems; Klärung ob Problem } \\
\text { intra- oder interpersonal bearbeitet werden soll: Klientin } \\
\text { soll zum Perspektivenwechsel angeregt werden, Ereignis soll } \\
\text { nicht aus eigener Sicht, sondern aus Perspektive der Mutter } \\
\text { Beschrieben werden }\end{array}$ \\
\hline
\end{tabular}

Tab. 2: Bewertung der beobachteten Interventionen: Vermutete Ziele zu Videosequenz 1 und 2 (Anmerkungen: Videosequenz 1 wurde zu EZ 1 gezeigt, Videosequenz 2 zu EZ 2.)

matter \& Tschacher (2016). In einem zweiten Durchgang wurden die Begründungen der Arbeitsgruppen mit der Beschreibung allgemeiner Wirkfaktoren aus der Literatur (Pfammatter \& Tschacher, 2012) mittels MAXQDA zugeordnet und anschliessend den übergeordneten Klassen kognitive Verarbeitung, Problembewältigung, emotionale Verarbeitung und Beziehung- und Motivationsaufbau zugeteilt.

Bei der Analyse der Bewertung der psychotherapeutischen Intervention aus dem Video ist aufgefallen, dass nicht alle Interventionen durch das PAP-S-RM abgebildet scheinen. Jede Gruppe nahm eine Beschreibung zur Mentalisierungsfähigkeit der Klientin vor. Nach Rücksprache mit Frau Koemeda-Lutz und durch die Diskussionsbeiträge am Theoriekolloquium zeigte sich, dass die Schnittstelle zwischen emotionalem Erleben und kognitiver Verarbeitung, die die Mentalisierungsbasierte Therapie auszeichnet, im Manual (noch) nicht abgebildet ist. Sie wurde als neue Kategorie unter dem Titel Selbstreflexivität/Mentalisierung erstellt. Pfammatter \& Tschacher (2012) benutzen diese Begrifflichkeit als eine Kategorie der allgemeinen Faktoren. Nach Aussage der Teilnehmenden wird sie von ihnen vor allem für diagnostische Zwecke verstanden. Während allen Forschungskolloquien, bei den Fragen nach der Bewertung, aber auch bei den alternativen Interventionen wurde das Setting von den Gruppen angesprochen. Daher wurde Setting ebenfalls als ergänzende Kategorie ausserhalb des PAP-S-RM aufgenommen.

\section{Ergebnisse}

Im ersten Teil von Fragebogen 1 wird nach den vermuteten Zielen der Therapeutin aus dem Video gefragt. Die von den einzelnen Gruppen formulierten Ziele zur Videosequenz 1 reichten von Beziehungsaufbau bis zu Diagnostik. Zur Videosequenz 2 wurde neben diagnostischen Frageklärungen vermutet, dass es das Ziel der Therapeutin ist, zwischen der Wahrnehmung der Klientin und der Mutter zu unterscheiden (vgl. Tab. 2). Die Arbeitsgruppe Prozessorientierte Psychotherapie hatte bei der ersten Sequenz ein Übertragungsgeschehen identifiziert, dem kein Ziel zuordenbar war.

Im zweiten Teil von Fragebogen 1 wurden die Arbeitsgruppen zu ihren alternativen Reaktionen anstelle derjenigen der Therapeutin befragt, also welche Interventionen sie mit welchem Ziel einsetzten würden. Auch hier geschah die PAP-S-RM-Zuordnung durch die Autorin der vorliegenden Arbeit. Für die Videosequenz 1 zielen die Interventionen der Gruppen unter anderem darauf $a b$, die Informationssammlung zu vertiefen, das affektive (und damit verbundene körperliche) Erleben sowie die Mentalisierung und Reflexionsfähigkeit der Klientin besser kennenzulernen. Die dazu vorgeschlagenen Interventionen dienen dementsprechend dazu, die emotionale und kognitive Verarbeitung der Klientin zu unterstützen. Für Videosequenz 2 wurden Interventionen mit den Zielen der weiteren Klärung auf emotionaler und kognitiver Ebene vorgeschlagen und auf die Bereiche Problembewältigung und Beziehungs- und Motivationsaufbau ausgeweitet.

Die konkreten Interventionen der Gruppen, die sie anstelle der Therapeutin bei der Klientin anwenden würden, wurden abermals mittels PAP-S-RM von der Autorin der vorliegenden Arbeit zugeordnet (vgl. Tab. 3). Hier zeigte sich, dass die Formulierungen der Gruppen zu einem grossen Teil den allgemeinen Faktoren zugeordnet werden konnten. Ihnen folgen die schulspezifischen Faktoren und 


\begin{tabular}{|l|c|c|c|c|c|c|c|c|}
\hline \multicolumn{1}{|c|}{ AGUST-Gruppe } & \multicolumn{2}{|c|}{$\begin{array}{c}\text { Allgemeine } \\
\text { Faktoren }\end{array}$} & $\begin{array}{c}\text { Schulspezifi- } \\
\text { sche Faktoren }\end{array}$ & \multicolumn{2}{c|}{$\begin{array}{c}\text { Spezifische } \\
\text { Faktoren }\end{array}$} & \multicolumn{2}{c|}{ Andere } \\
\hline & EZ 1 & EZ 2 & EZ 1 & EZ 2 & EZ 1 & EZ 2 & EZ 1 & EZ 2 \\
\hline Gestaltpsychotherapie & 0 & 3 & 4 & 3 & 0 & 0 & 0 & 0 \\
\hline Humanistische Psychotherapie & 1 & 4 & 2 & 1 & 1 & 0 & 1 & 3 \\
\hline Jung'sche Psychoanalyse & 1 & 2 & 0 & 2 & 0 & 2 & 0 & 0 \\
\hline Prozessorientierte Psychotherapie & 3 & 1 & 0 & 0 & 1 & 1 & 0 & 0 \\
\hline Freud'sche Psychoanalyse & 1 & 4 & 2 & 0 & 0 & 0 & 0 & 0 \\
\hline Summe & 6 & 13 & 8 & 6 & 2 & 3 & 1 & 3 \\
\hline
\end{tabular}

Tab. 3: Konkrete alternative Reaktionen: PAP-S-RM-Zuordnung über EZ 1 und 2

mit Abstand die spezifischen Faktoren. Betrachtet man die Anzahl der allgemeinen Faktoren (20) vergleichend mit schulspezifischen und spezifischen Faktoren $(14+5)$ ergibt sich eine fast gleichmässige Verteilung der konkreten Interventionen.

In Tabelle 4 sind die PAP-S-RM-Zuordnungen der konkreten Interventionsvorschläge im Detail zu sehen. $\mathrm{Zu}$ Klarifizieren (Nr. 55) als sowohl allgemeiner als auch schulspezifischer Faktor wurden von jeder Gruppe Formulierungen erarbeitet, zu Arbeit am subjektiven Empfinden (Nr. 8) die meisten Alternativinterventionen erstellt, auch wenn diese Kategorie nicht von allen Gruppen verwendet wurde. Andere Aussagen, die nicht mit dem Manual eingeordnet werden konnten, waren Schweigen als Intervention und Selbstreflexivität/Mentalisierung.

Im Anschluss an den Vorschlag der konkreten Interventionen wurden die teilnehmenden Gruppen darum gebeten, die Auswahl ihrer Alternativintervention zu begründen. Als Ordnungssystem für diese Begründungen haben sich die Klassen allgemeiner Wirkfaktoren (Pfammatter \& Tschacher, 2016) angeboten, die die Forschenden 2012 zusammengestellt haben. Die Verwendung über beide Forschungskolloquien ist anhand Abbildung 2, die Verwendung innerhalb der Arbeitsgruppen anhand Abbildung 3 zu sehen.

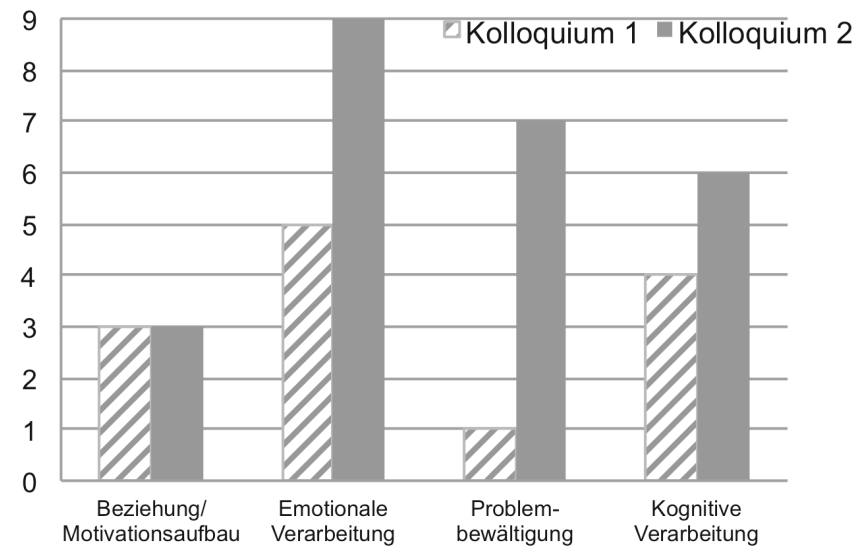

Abb. 2: Alternative Interventionen: Cluster-Zuordnung der Begründungen (nach Pfammatter \& Tschacher, 2016; ̈̈ber EZ 1 und 2)

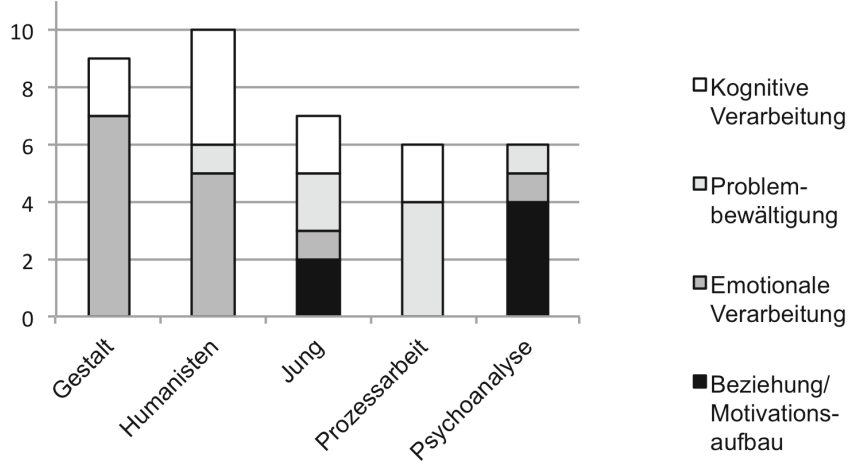

Abb. 3: Alternative Interventionen: Cluster-Zuordnung der Begründungen (nach Pfammatter \& Tschacher, 2016)

Damit konnte das Cluster Emotionale Verarbeitung mit $37 \%$ am häufigsten zur Begründung der alternativen Interventionen verwendet werden. Es beinhaltet die zugeordneten Wirkfaktoren Affektregulation, Korrektive emotionale Erfahrung und Affektives Erleben. Das Cluster Kognitive Verarbeitung liegt an zweiter Stelle mit 26\% Anteil an den Begründungen der Gruppen und umfasst die Wirkfaktoren Klärung und Einsicht, Neue Narration des Selbst (zu gleichen Teilen) und Mentalisierung und Selbstreflexivität. Problembewältigung wurde im Vergleich für $21 \%$ aller Begründungen der alternativen Interventionen zugeordnet. Diesem Cluster gehören die Faktoren Problemaktualisierung, Erhöhung der Selbstwirksamkeitserwartung, Ressourcenaktivierung und Erklärungssystem an. Das Cluster Beziehung/Motivationsaufbau wurde für $16 \%$ der Angaben verwendet und kommt bei den psychoanalytischen Gruppen zum Zuge. Sie begründeten insgesamt sechs ihrer alternativen Reaktionen damit. Die Untergruppen dieses Clusters sind Abschwächung sozialer Isolation und Therapiebeziehung.

Zum dritten Kolloquium, dem Theoriekolloquium, wurden die Teilnehmenden des AGUST-Projekts zum ganzen Film befragt. Dafür kam Fragebogen 2 zum Einsatz mit den Schwerpunkten: Probleme der Klientin, Abbildung des schulspezifischen Hintergrundes und Gegenübertragungsgeschehen, die im Folgenden vorgestellt 


\begin{tabular}{|c|c|c|c|c|c|c|c|c|c|c|c|}
\hline \multirow[t]{3}{*}{ AGUST-Arbeitsgruppe } & \multicolumn{2}{|c|}{$\begin{array}{l}\text { Gestaltpsy- } \\
\text { chotherapie }\end{array}$} & \multicolumn{2}{|c|}{$\begin{array}{l}\text { Humanisti- } \\
\text { sche Psycho- } \\
\text { therapie }\end{array}$} & \multicolumn{2}{|c|}{$\begin{array}{l}\text { Jung'sche } \\
\text { Psycho- } \\
\text { analyse }\end{array}$} & \multicolumn{2}{|c|}{$\begin{array}{l}\text { Prozessorien- } \\
\text { tierte Psycho- } \\
\text { therapie }\end{array}$} & \multicolumn{2}{|c|}{$\begin{array}{l}\text { Freud'sche } \\
\text { Psycho- } \\
\text { analyse }\end{array}$} & $S$ \\
\hline & \multicolumn{2}{|c|}{ EZ } & \multicolumn{2}{|c|}{ EZ } & \multicolumn{2}{|c|}{ EZ } & \multicolumn{2}{|c|}{ EZ } & \multicolumn{2}{|c|}{ EZ } & \\
\hline & 1 & 2 & 1 & 2 & 1 & 2 & 1 & 2 & 1 & 2 & \\
\hline \multicolumn{12}{|l|}{ nicht im PAP-S-RM abgebildet } \\
\hline Selbstreflexivität/Mentalisierung & & & & 3 & & & & & & & 3 \\
\hline Schweigen & & & 1 & & & & & & & & 1 \\
\hline
\end{tabular}

Allgemeine Faktoren

\begin{tabular}{|c|l|c|c|c|c|c|c|c|c|c|c|}
\hline 8 & $\begin{array}{l}\text { Arbeit am subj. Erleben/ } \\
\text { Empfinden }\end{array}$ & & & 1 & 2 & 1 & 2 & & & 1 & 8 \\
\hline 19 & $\begin{array}{l}\text { Aufmerksamkeitslenkung } \\
\text { auf akt. Emotionen/Gefühle }\end{array}$ & & & & & & & & & & 1 \\
\hline 31 & Empathie & & 2 & & & & & 2 & & & \\
\hline 55 & Klarifizieren & & 1 & 2 & & & & & 1 & 1 & 4 \\
\hline 88 & $\begin{array}{l}\text { Therapiekontakt/therap. } \\
\text { Beziehung }\end{array}$ & & & & & & & & & 2 & 2 \\
\hline
\end{tabular}

Spezifische Faktoren

\begin{tabular}{|c|l|l|l|l|l|l|l|l|l|l|l|}
\hline 21 & $\begin{array}{l}\text { Bearbeitung von Verhaltens- } \\
\text { mustern/Überzeugungen }\end{array}$ & & & & & 1 & & 1 \\
\hline 38 & $\begin{array}{l}\text { Fragen zu Möglichkeits- } \\
\text { konstruktionen }\end{array}$ & & & 1 & & & & & & & \\
\hline 56 & $\begin{array}{l}\text { Körperebene bewusst } \\
\text { machen }\end{array}$ & & & & & & 1 & & 1 & & \\
\hline 76 & Rollenspiel-Initiierung & & & & & & & & 1 & & \\
\hline
\end{tabular}

Schulspezifische Faktoren

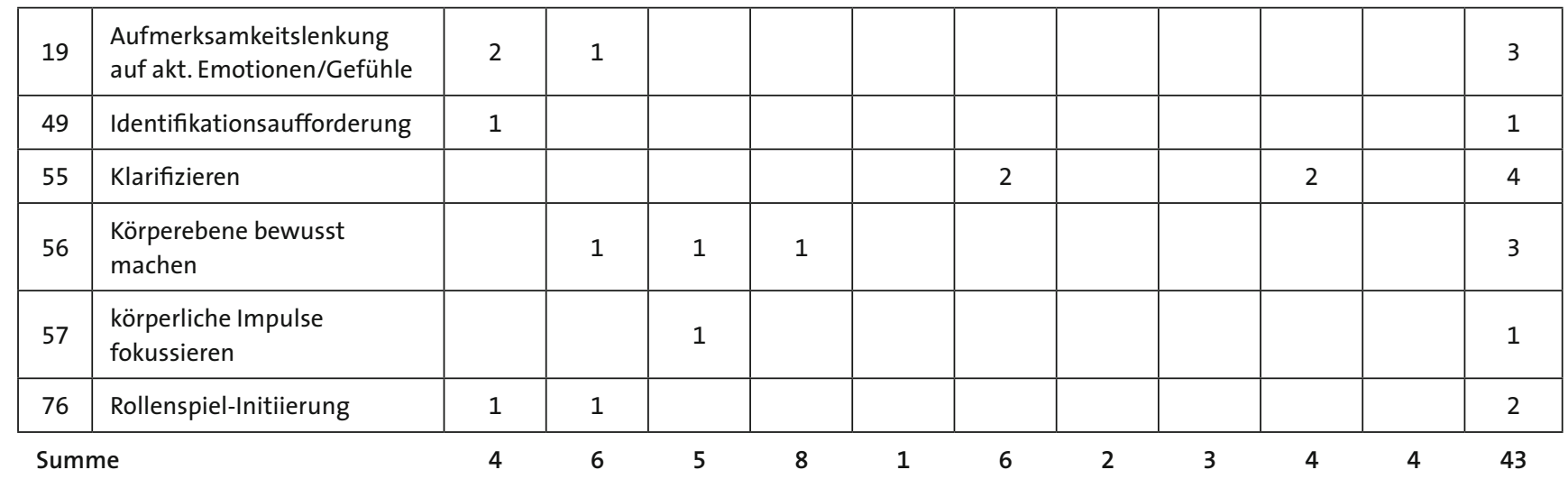

Tab. 4: Konkrete alternative Reaktionen: Detaillierte Verteilung der PAP-S-RM-Zuordnung über EZ 1 und 2

werden. Die von den einzelnen Gruppen vermuteten Problembereiche der Klientin umfassen: Ablösungsprobleme, Angst und Einsamkeit sowie Selbstwertprobleme im weitesten Sinne.

Die Interventionen der Arbeitsgruppen, mit denen sie ihre schultheoretischen Konzepte abbilden möchten, konnten gleichermassen den schulspezifischen Faktoren wie den allgemeinen Faktoren zugeordnet werden (s. Tab. 5). Ausnahmen bildeten dabei jeweils ein spezifischer Interventionsvorschlag der Gruppen Prozessorien- tierte Psychotherapie (bedingungsfreie positive Beachtung) und Freud'sche Psychoanalyse (positive Verstärkung). Nicht alle von den ProbandInnen vorgeschlagenen Interventionen konnten codiert werden. Elf der 38 Interventionsabbildungen konnten nicht mittels PAP-S-RM zugeordnet werden (beispielsweise: Thema nicht vorgeben, Mentalisierung, Schwerpunkt setzen, Platz lassen, Schweigen), was alle Arbeitsgruppen betraf.

Nachfolgend werden die Ergebnisse der qualitativen Auswertung zu den jeweiligen Arbeitsgruppen zusam- 


\begin{tabular}{|l|c|c|c|}
\hline \multicolumn{1}{|c|}{ AGUST-Gruppe } & Allgemeine Faktoren & Schulspezifische Faktoren & Spezifische Faktoren \\
\hline & EZ 3 & EZ 3 & EZ 3 \\
\hline Gestaltpsychotherapie & 2 & 3 & 0 \\
\hline Humanistische Psychotherapie & 3 & 3 & 0 \\
\hline Jung'sche Psychoanalyse & 2 & 2 & 0 \\
\hline Prozessorientierte Psychotherapie & 0 & 1 & 1 \\
\hline Freud'sche Psychoanalyse & 5 & 3 & 1 \\
\hline Summe & 12 & 12 & 2 \\
\hline
\end{tabular}

Tab. 5: Abbildung des schulspezifischen Therapiehintergrundes: PAP-S-RM-Zuordnung

mengefasst. In der Gestaltpsychotherapie ist ein wichtiger Grundsatz auf Interventionsebene, davon auszugehen, dass das zu bearbeitende Thema auftaucht, wenn es bearbeitet werden will. Das Ziel für Karina sieht der Vertreter der Gestaltpsychotherapie unter anderem darin, ihre Bedürfnisse und Emotionen gegenüber der Umgebung auszudrücken. In der Arbeitsgruppe Humanistische Psychotherapie können die Untergruppen Gespräch, Focusing, Körper (GFK) und Existenzanalyse (EA) voneinander unterschieden werden. Während die VertreterInnen des Ausbildungsinstituts GFK einen Mini-Focusing-Prozess abbilden möchten, sehen die ExistenzanalytikerInnen die emotionale Auseinandersetzung und deren Verankerung bei der Klientin als Ziel. Das verfolgen sie mit verschiedenen Angeboten, wie zum Beispiel durch Stellungnahme (EA) oder Herstellung des Körperbezuges (GFK). Nicht gelebte Anteile ins Leben treten lassen - das zielt die Arbeitsgruppe Jung'sche Psychoanalyse an und bildet unter anderem damit ihr schultheoretisches Konzept ab. Dafür würden sie Interventionen anbieten, die die Gefühlswelt beleuchten und die Mentalisierung unterstützen. Die Arbeitsgruppe Prozessorientierte Psychotherapie wählte zur Abbildung ihres schulspezifischen Zieles die bewusstere Erfahrung von identitätsfernen Persönlichkeitsanteilen. Dieses Ziel erreichen sie unter anderem mit dem Erlebbar-machen von Selbstermächtigung, Bewegung und Rollenspielen. Wollten die Vertreter der Freud'schen Psychoanalyse ihr schultheoretisches Konzept darstellen, dann wäre ein Ziel, der Klientin eine alternative Erfahrung anzubieten, indem sie sich verstanden fühlt. Dafür würden sie unter anderem die Klientin darin unterstützen, Platz einzunehmen, und versuchen, sie zu entlasten.

Es wurden unterschiedliche Gegenübertragungsgefühle von den Gruppen beschrieben, mit Überschneidungen. Die Gruppe Gestaltpsychotherapie hatte Mitleid mit der Klientin und sieht sie nicht abgeholt in der Anerkennung die sie braucht. Die Gruppe der Humanistische Psychotherapie beschrieb ihren Eindruck von der exemplarischen Therapiestunde im Video für die Klientin als Reinszenierung dessen, was sie mit der Mutter erlebt. Teilweise war auch Ärger über die Anpassung der Patientin spürbar. Die Gruppe Jung'sche Psychoanalyse formulierte das Gegenübertragungsgeschehen als der Klientin zugewandt, parteiisch. Für sie entstanden Gefühle wie Einen-Schutzraum-geben-wollen.
In der interessiert zugewandten, bestätigenden und fördernden Elternrolle sahen sich die Mitglieder der Prozessorientierten Psychotherapie der Klientin gegenüber. Die Gruppe Freud'sche Psychoanalyse beschrieb den Wechsel zwischen konkordanter und komplementärer Gegenübertragung. Der Klientin gegenüber äusserten sie sehr viel Mitgefühl bis hin zu Mitleid, aber auch ein Gefühl von Langeweile, da sie wenig Emotionalität ins Gespräch einbringt.

\section{Diskussion}

Das AGUST-Projekt der ASP sollte die Frage klären, welche Gemeinsamkeiten und Unterschiede im Rahmen einer Videoanalyse PsychotherapeutInnen verschiedener schultheoretischer Ausrichtung bei der Beurteilung einer (fremden) Therapiesitzung und bei der Auswahl (eigener) Alternativinterventionen haben. Gemeinsamkeiten zeigten sich bei der Bewertung des Videos, das mentalisierungsbasierte Vorgehen wurde von allen Arbeitsgruppen identifiziert, auch wenn die Mentalisierungsbasierte Therapie den meisten nicht bekannt war. Auch bzgl. der alternativen Interventionsvorschläge gab es Gemeinsamkeiten: Es wurden vor allem Interventionen zur Unterstützung der emotionalen und kognitiven Verarbeitung verwendet. Die alternativen Ziele lassen sich zusammenfassen unter der Einnahme sowohl einer Klärungs- als auch Bewältigungsperspektive der PsychotherapeutInnen im Falle von der Klientin des Videos. Bei der Zuordnung mittels PAP-S-RM wurde gruppenübergreifend das Klarifizieren genannt.

Bei den Begründungen der jeweiligen Alternativinterventionen wurde vor allem die Unterstützung der emotionalen und kognitiven Verarbeitung angegeben, gefolgt von Problembewältigung. Die Begründungen scheinen unter diesen Aspekten schultypisch verlaufen zu sein. Bei der Problembeschreibungen zur Klientin gab es Gemeinsamkeiten und Unterschiede, zum Teil überschnitten sich die Inhalte, sprachlich waren die einzelnen Ausrichtungen jedoch unterscheidbar. Auch zur Abbildung der eigenen Orientierung fanden sich schultypische Formulierungen. Mittels PAP-S-RM waren jedoch auch Unterschiede erkennbar: Von allen Gruppen wurden auch unspezifische Interventionen gewählt, die also keine schulspezifischen Techniken enthalten und allgemein 
wirksam sind. Die Kombination bzw. das Zusammenspiel allgemeiner und schulspezifischer bzw. spezifischer Faktoren schien für die Arbeitsgruppen am Geeignetsten, um ihre therapeutischen Ziele bei Karina zu erreichen

Im AGUST-Projekt zeigen sich bei der Befragung von VertreterInnen verschiedener schultheoretischer Konzepte sowohl Gemeinsamkeiten als auch Unterschieden bei der Bewertung einer exemplarischen Therapiestunde sowie der Erstellung von Alternativinterventionen. Die Erklärungssysteme der einzelnen schultheoretischen Ausrichtungen unterscheiden sich vor allem in den sprachlichen Formulierungen, auf konkreter Anwendungsebene wählten die Arbeitsgruppen ähnliche Vorgehensweisen. Deutlich wurde, dass die Teilnehmenden allgemein wirksame Interventionen genauso einsetzen wie schulspezifische und spezifisch wirksame Interventionen, um ihre therapeutischen Ziele zu erreichen. Das bestätigt, dass in psychotherapeutischen Behandlungen nicht nur schultheoretisches Wissen angewendet wird, sondern gleichzeitig auch allgemeine, die Interaktions- und Kommunikationsprozesse fördernde Strategien gewählt werden.

\section{Literatur}

Ambühl, H. \& Orlinsky, D. (1997). Zum Einfluss der theoretischen Orientierung auf die psychotherapeutische Praxis. Psychotherapeut, 5, 290-298.

Goldfried, M.R. (1980). Toward the delineation of therapeutic change principles. American Psychologist, 35, 991-999. https://doi.org/ 10.1037/0003-066X.35.11.991

Grawe, K. (2000). Psychologische Therapie. Göttingen: Hogrefe.

Hill, C.E. \& O'Grady K.E. (1985). List of therapist intentions illustrated in a case study and with therapists of varying theoretical orientations. Journal of Counceling Psychology, 32(1), 3-22.

Mayring, P. (2010). Qualitative Inhaltsanalyse. In G. Mey \& K. Mruck (Hrsg.), Handbuch qualitative Forschung in der Psychologie (S. 601-613). Wiesbaden: Springer.

Mayring, P. (2015). Qualitative Inhaltsanalyse. Grundlagen und Techniken. Weinheim, Basel: Beltz.

Mayring, P. (2016). Einführung in die qualitative Sozialforschung. Weinheim: Beltz.

Norcross, J.C. \& Lambert, M.J. (2018). Psychotherapy relationships that works III. Psychotherapy, 55(4), 303-315. https://doi. org/10.1037/pst0000193

Pfammatter, M. \& Tschacher, W. (2012). Wirkfaktoren in der Psychotherapie - eine Übersicht und Standortbestimmung. Zeitschrift für Psychiatrie, Psychologie und Psychotherapie, 60(1), 67-76. https:// doi.10.1024/1661-4747/a000099

Pfammatter, M. \& Tschacher, W. (2016). Klassen allgemeiner Wirkfaktoren der Psychotherapie und ihr Zusammenhang mit Therapietechniken. Zeitschrift für Klinische Psychologie und Psychotherapie, 45(1), 1-13. https://doi.10.1026/1616-3443/a000331

Strauss, B. \& Schauenburg, H. (Hrsg.). (2017). Bindung in Psychologie und Medizin. Grundlagen, Klinik und Forschung - Ein Handbuch. Stuttgart: Kohlhammer.

Strotzka, H. (1969). Psychotherapie und soziale Sicherheit. Bern: Huber.

Tschuschke, V., Koemeda-Lutz, M. \& Schlegel, M. (2014). PAP-SRating-Manual. Rating Manual zur objektiven Einschätzung therapeutischer Interventionen von Psychotherapeuten unterschiedlicher schultheoretischer Konzepte. Zürich: Schweizer Charta für Psychotherapie. http://psychotherapiecharta.ch.sokrates.ch-meta.net/ charta/fdownloads/Neueste\%20Downloads/Neue\%20Downloads/ rating-manual-paps-web.pdf
Tschuschke, V. (2016). Therapeutische Interventionstechniken: Zur Bedeutung der Konzepttreue in der Psychotherapie. In A.v. Wyl, V. Tschuschke, A. Crameri, M. Koemda-Lutz \& P. Schulthess (Hrsg.), Was wirkt in der Psychotherapie? (S. 75-85). Gießen: Psychosozial-Verlag.

v. Wyl, A., Tschuschke, V., Crameri, A., Koemeda-Lutz, M. \& Schulthess, P. (Hrsg.). (2016). Was wirkt in der Psychotherapie? Ergebnisse der Praxisstudie ambulante Psychotherapie zu 10 unterschiedlichen Verfahren. Gießen: Psychosozial-Verlag.

Wampold, B.E., Imel Z.E. \& Flückiger C. (2018). Die Psychotherapie-Debatte. Was Psychotherapie wirksam macht. Bern: Hogrefe.

Wirtz, M. A. (2016). Forschungsmethoden und Evaluation. In M. A. Wirtz (Hrsg.), Dorsch - Lexikon der Psychologie. https:// portal.hogrefe.com/dorsch/intervention

Wissenschaftlicher Beirat Psychotherapie [WBP] (2008). Glossar zu wiederkehrenden Begriffen im Zusammenhang mit den Stellungnahmen des Wissenschaftlichen Beirats Psychotherapie. http://www.wbpsychotherapie.de/stellungnahmen/sonstige-stellungnahmen-und-veroeffentlichungen/glossar

\section{The AGUST-Project. Analysis of similarities and differences in school-specific psychotherapy methods}

Abstract: The science commission of the Association of Swiss Psychotherapists (ASP) initiated the AGUST-Project to figure out commonalities and differences at the application of psychotherapeutic interventions. 24 representatives of eight therapy schools analysed in five working groups an APA video of a therapeutic session and answered questions to their own practice. Between September 2018 and January 2019 three science colloquium took place. On the exampel of the client of the video, the engaged therapists rated the shown interventions as they formulared alternative suggestions for interventions and goals in line of their therapy schools and explained their rationale for that. In the center of the research was the concrete approach of the particular school. The analysis shows commonalities and differences between the single psychotherapeutic orientations. In the video rating, in the suggestions of alternative interventions and their classification by the PAP-S-Rating-Manual the working groups showed less differences as for the rationale of their alternative interventions, the description of the client problems and terms they used to illustrate their specific backround.

Keywords: psychotherapy research, psychotherapeutic interventions, efficacy factors, theoretical orientation

\section{Il progetto AGUST. Analisi di somiglianze e differenze delle procedure di psicoterapia specifiche della scuola.}

Riassunto: La Commissione scientifica della Charta svizzera per la psicoterapia (WiKo), un organo dell'Associazione degli psicoterapeuti svizzeri (ASP), ha implementato con AGUST il progetto per studiare i punti in comune e le differenze nell'uso degli interventi di psicoterapia. Con questo scopo, sono stati intervistati ventiquattro rappresentanti di otto diverse scuole di psicoterapia su un video didattico APA. In tre colloqui di ricerca, che si sono svolti tra settembre 2018 e gennaio 2019, essi hanno valutato il video in gruppi di lavoro. Utilizzando l'esempio della paziente del video, nei gruppi sono stati anche elaborati suggerimenti di intervento psicoterapeutico alternativo e obiettivi terapeutici e sono state raccolte le relative motivazioni. Il punto focale dell'interesse della ricerca era l'approccio concreto delle 
singole scuole. Vi erano somiglianze e differenze tra i singoli gruppi di lavoro. Nel valutare il video, le proposte di reazione alternative e la loro associazione con il manuale di valutazione PAP-S, i gruppi differivano di meno rispetto alla motivazione degli interventi alternativi, alla loro descrizione dei problemi della paziente del video e alla terminologia per l'illustrazione del loro background specifico della scuola.

Voci: ricerca in psicoterapia, interventi psicoterapeutici, fattori, scuole di psicoterapia

\section{Die Autorlnnen}

Cornelia Stegmann studiert an der ZHAW, Departement Angewandte Psychologie.

Prof. Dr. Rosmarie Barwinski, MSc Katrin Hartmann, Dr. Mario Schlegel und Prof. Dr. Agnes von Wyl sind Mitglieder der WiKo der ASP.

\section{Kontakt}

E-Mail: cornelia.stegmann@icloud.com 